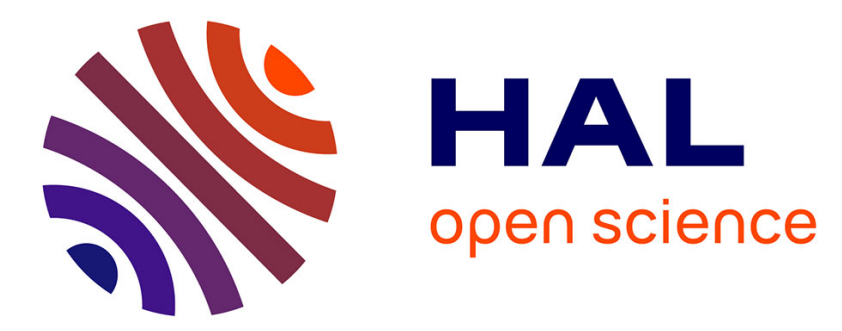

\title{
On the global existence of weak solution for a multiphasic incompressible fluid model with Korteweg stress
}

Caterina Calgaro, Meriem Ezzoug, Ezzeddine Zahrouni

\section{To cite this version:}

Caterina Calgaro, Meriem Ezzoug, Ezzeddine Zahrouni. On the global existence of weak solution for a multiphasic incompressible fluid model with Korteweg stress. Mathematical Methods in the Applied Sciences, 2017, 10.1002/mma.3969 . hal-01388718

\section{HAL Id: hal-01388718 \\ https://hal.science/hal-01388718}

Submitted on 27 Oct 2016

HAL is a multi-disciplinary open access archive for the deposit and dissemination of scientific research documents, whether they are published or not. The documents may come from teaching and research institutions in France or abroad, or from public or private research centers.
L'archive ouverte pluridisciplinaire HAL, est destinée au dépôt et à la diffusion de documents scientifiques de niveau recherche, publiés ou non, émanant des établissements d'enseignement et de recherche français ou étrangers, des laboratoires publics ou privés. 


\title{
On the global existence of weak solution for a multiphasic incompressible fluid model with Korteweg stress
}

\author{
Caterina CAlgaro ${ }^{*} \quad$ Meriem Ezzoug $^{\dagger} \quad$ Ezzeddine Zahrouni ${ }^{\ddagger}$
}

March 26, 2016

\begin{abstract}
In this paper, we study a multiphasic incompressible fluid model, called the Kazhikhov-Smagulov model, with a particular viscous stress tensor, introduced by Bresch and co-authors, and a specific diffusive interface term introduced for the first time by Korteweg in 1901. We prove that this model is globally well posed in a $3 \mathrm{D}$ bounded domain.
\end{abstract}

Keywords :Mixture theory; Kazhikhov-Smagulov model; Korteweg model; global existence result.

\section{Introduction}

In this paper, we are interested in the study of a system of PDEs describing the evolution of mixture flows. Let $\Omega$ be a bounded open set in $\mathbb{R}^{3}$ with boundary $\Gamma$ that is regular enough and let $\boldsymbol{n}$ be the outward unit normal on the boundary $\Gamma$. We denote by $[0, T]$ the time interval, for $T>0$. We consider the following system of PDEs derived from the compressible Navier-Stokes equations with a Korteweg stress tensor. The closure of the system is given by a Fick's law which relates the velocity to derivatives of the density. This system, that we call the Kazhikhov-Smagulov-Korteweg (KSK) model, reads

$$
\left\{\begin{array}{l}
\rho\left(\partial_{t} \boldsymbol{u}+(\boldsymbol{u} \cdot \nabla) \boldsymbol{u}\right)-\lambda(\nabla \rho \cdot \nabla) \boldsymbol{u}-\frac{\lambda}{2} \operatorname{div}\left(\rho \nabla \boldsymbol{u}-\rho \nabla \boldsymbol{u}^{T}\right)+\nabla P=\rho \boldsymbol{g}-\kappa \Delta \rho \nabla \rho, \\
\partial_{t} \rho+\operatorname{div}(\rho \boldsymbol{u})=\lambda \Delta \rho, \\
\operatorname{div} \boldsymbol{u}=0 .
\end{array}\right.
$$

With the notations $\mathcal{Q}_{T}=(0, T) \times \Omega$ and $\Sigma=(0, T) \times \Gamma$, the unknowns for this model are $\rho: \mathcal{Q}_{T} \rightarrow \mathbb{R}$ the density of the fluid, $\boldsymbol{u}: \mathcal{Q}_{T} \rightarrow \mathbb{R}^{3}$ the mean volume velocity field and $P: \mathcal{Q}_{T} \rightarrow \mathbb{R}$ the pressure of the fluid (a modified pressure). Moreover, $\boldsymbol{g}$ stands for the gravity acceleration (but it can include further external forces) and the parameters $\lambda>0$ and $\kappa>0$ represent mass diffusion cœefficient and Korteweg's constant, respectively.

The KSK model (1) is completed with the following boundary and initial conditions

$$
\begin{gathered}
\boldsymbol{u}(t, \boldsymbol{x})=0, \quad \frac{\partial \rho}{\partial \boldsymbol{n}}(t, \boldsymbol{x})=0, \quad(t, \boldsymbol{x}) \in \Sigma, \\
\boldsymbol{u}(0, \boldsymbol{x})=\boldsymbol{u}_{0}(\boldsymbol{x}), \quad \rho(0, \boldsymbol{x})=\rho_{0}(\boldsymbol{x}), \quad \boldsymbol{x} \in \Omega,
\end{gathered}
$$

\footnotetext{
${ }^{*}$ Univ. Lille, CNRS, UMR 8524 - Laboratoire Paul Painlevé, F-59000 Lille, France. caterina.calgaro@math.univ-lille1.fr

${ }^{\dagger}$ Unité de Recherche, Multifractales et Ondelettes, FSM, University of Monastir. Tunisia. meriemezzoug@yahoo.fr

${ }^{\ddagger}$ FSEGN, University of Carthage, 8000 Nabeul, Tunisia. ezzeddine.zahrouni@fsm.rnu.tn
} 
with the compatibility condition $\operatorname{div} \boldsymbol{u}_{0}=0$, where $\rho_{0}: \Omega \rightarrow \mathbb{R}$ and $\boldsymbol{u}_{0}: \Omega \rightarrow \mathbb{R}^{3}$ are given functions. Throughout this work, we assume the hypothesis

$$
0<m \leq \rho_{0}(\boldsymbol{x}) \leq M<+\infty, \quad \boldsymbol{x} \in \Omega .
$$

Let us mention some known results about the Kazhikhov-Smagulov model without the Korteweg stress tensor. Let us also mention other results about the incompressible Navier-Stokes equations with a Korteweg stress tensor, coupled with a convection or a convection-diffusion equation for the density of the mixture.

First, we take $\kappa=0$. In [4], Bresch et al. consider the problem (1) (without Korteweg term), (2) and (3). They prove the global existence of weak solution without assuming small data and without any assumption on the diffusivity $\lambda$. In $[1,3]$, the equation $(1)_{1}$ is replaced by

$$
\rho\left(\partial_{t} \boldsymbol{u}+(\boldsymbol{u} \cdot \nabla) \boldsymbol{u}\right)-\lambda(\nabla \rho \cdot \nabla) \boldsymbol{u}-\lambda(\boldsymbol{u} \cdot \nabla) \nabla \rho-\mu \boldsymbol{\Delta} \boldsymbol{u}+\nabla P+\frac{\lambda^{2}}{\rho}\left(\Delta \rho \nabla \rho+(\nabla \rho \cdot \nabla) \nabla \rho-\frac{|\nabla \rho|^{2}}{\rho} \nabla \rho\right)=\rho \boldsymbol{g} .
$$

In [1], Antontsev et al. study the problem (5) - (1) $)_{2}-(1)_{3},(2)$ and (3) without $\mathcal{O}\left(\lambda^{2}\right)$ terms in (5). Under assumption (4) and if constants $\lambda, \mu, m, M$ are such that $\lambda<2 \mu(M-m)^{-1}$, they prove that there exists at least one weak solution of the problem, given $\boldsymbol{u}_{0} \in \mathbf{H}, \rho_{0} \in H^{1}(\Omega)$ and $\boldsymbol{g} \in L^{2}\left(0, T ; \mathbf{L}^{2}(\Omega)\right)$. If additionally $\boldsymbol{u}_{0} \in \mathbf{V}$, then the problem has a unique strong solution locally in time. This solution is global in the two-dimensional case. In [3], Beirão da Veiga considers the same problem with all the terms in (5) and he proves the existence of a unique local solution for arbitrary initial data and external forces and the existence of a unique global strong solution for small initial data and external forces. In particular, if $\boldsymbol{g}=0$, the solution decay exponentially in time to the equilibrium solution with zero velocity field.

Second, we consider $\kappa>0$. In [13], Kostin et al. study the mathematical model consisting of a convectiondiffusion equation for the concentration and homogeneous Navier-Stokes equations with the Korteweg stress. They prove the global existence and uniqueness of the solution for the initial-boundary value problem in a two-dimensional bounded domain and its asymptotic for large time. In [17], Sy et al. take the homogeneous Navier-Stokes equations with the Korteweg stress coupled with the mass conservation equation. Their result, given in a three-dimensional bounded domain, concerns the existence of a unique local solution for any initial data and the existence of a unique global solution for small initial data.

The aim of this work is to prove the existence of a global in time weak solution (see Definition 3.1) of the Kazhikhov-Smagulov-Korteweg model (1) for arbitrary initial data and external force field. Our main result reads:

Theorem 1.1 Let $\boldsymbol{u}_{0} \in \boldsymbol{H}, \rho_{0} \in H^{1}(\Omega)$ satisfy (4), $T>0$ and $\boldsymbol{g} \in L^{2}\left(0, T ; \boldsymbol{L}^{2}(\Omega)\right)$. Then there exists a weak solution $(\boldsymbol{u}, \rho)$ of (1) global in time such that

$$
\begin{aligned}
& \boldsymbol{u} \in L^{\infty}(0, T ; \boldsymbol{H}) \cap L^{2}(0, T ; \boldsymbol{V}), \\
& \rho \in L^{\infty}\left(0, T ; H^{1}(\Omega)\right) \cap L^{2}\left(0, T ; H_{N}^{2}\right),
\end{aligned}
$$

with finite and uniformly bounded energy such that $\forall t \leq T$,

$$
\begin{aligned}
& \|\sqrt{\rho(t)} \boldsymbol{u}(t)\|_{L^{2}(\Omega)}^{2}+\kappa\|\nabla \rho(t)\|_{L^{2}(\Omega)}^{2}+\int_{0}^{t}\left(\frac{\lambda m}{4}\|\nabla \boldsymbol{u}(s)\|_{L^{2}(\Omega)}^{2}+2 \kappa \lambda\|\Delta \rho(s)\|_{L^{2}(\Omega)}^{2}\right) d s \\
& \leq\left\|\sqrt{\rho_{0}} \boldsymbol{u}_{0}\right\|_{L^{2}(\Omega)}^{2}+\kappa\left\|\nabla \rho_{0}\right\|_{L^{2}(\Omega)}^{2}+\frac{C M^{2}}{\lambda m} \int_{0}^{T}\|\boldsymbol{g}(s)\|_{L^{2}(\Omega)}^{2} d s .
\end{aligned}
$$

The outline of the paper is as follows. In section 2 we explain the derivation of the KSK model (1). In section 3 we present the functional setting. In section 4 we recall some preliminary results and in section 5 we prove the theorem 1.1 . 


\section{Modeling of the KSK model}

In this section we explain the derivation of the Kazhikhov-Smagulov-Korteweg model (1). The mixture of two fluids is described by the density $\rho(t, \boldsymbol{x}) \geq 0$, the mass velocity field $\boldsymbol{v}(t, \boldsymbol{x})$ and the pressure $p(t, \boldsymbol{x})$, depending on the time and space variables $(t, \boldsymbol{x}) \in[0, T] \times \Omega$. Joseph [10], Galdi et al. [9] and Joseph et al. [11] have developed a theory of non-solenoidal velocity effects and Korteweg stresses in a simple mixture of incompressible liquids. According to these authors, we consider the Korteweg equations for generalized incompressible fluids whose density and volume change with the concentration of one component in the binary mixture, but not with the pressure. This theory may be framed in terms of a mass velocity field $\boldsymbol{v}$ which is not solenoidal $(\operatorname{div} \boldsymbol{v} \neq 0$ ), and a volume velocity field $\boldsymbol{u}$ which is solenoidal ( $\operatorname{div} \boldsymbol{u}=0$ ). Assuming that each fluid is incompressible, the mass density is conserved in the absence of diffusion. The theory of Korteweg, introduced in [12] following earlier work by Van der Waals, considers the possibility that motions can be driven by additional stresses associated with gradients of density and gradients of the concentration of species. Dynamical effects which mimic surface tension can arise in thin mixing layers where the gradients of composition are large. It is worth pointing out that similar theories based on thermodynamic arguments are introduced by Brenner [2] (see also the references therein). The author emphasizes the assumption that density gradients give rise to stresses, even when these stresses are induced in single component liquids by temperature gradients.

On one hand, in order to model the fluid capillarity effects, Korteweg introduced in the usual compressible fluid model a specific stress tensor which depends on density derivatives. Following the rigorous formulation introduced by Dunn and Serrin [7] (see also Bresch et al. [5]) and neglecting thermal fluctuations, the model reads

$$
\left\{\begin{array}{l}
\partial_{t} \rho+\operatorname{div}(\rho \boldsymbol{v})=0, \\
\partial_{t}(\rho \boldsymbol{v})+\operatorname{div}(\rho \boldsymbol{v} \otimes \boldsymbol{v})=\rho \boldsymbol{g}+\operatorname{div}(\boldsymbol{S}+\boldsymbol{K}),
\end{array}\right.
$$

with the viscous stress tensor $\boldsymbol{S}$ and the Korteweg stress tensor $\boldsymbol{K}$ given by:

$$
\left\{\begin{array}{l}
\boldsymbol{S}=(\nu \operatorname{div} \boldsymbol{v}-p) \boldsymbol{I}+2 \mu \boldsymbol{D}(\boldsymbol{v}), \\
\boldsymbol{K}=\left(\alpha \Delta \rho+\beta|\nabla \rho|^{2}\right) \boldsymbol{I}+\delta(\nabla \rho \otimes \nabla \rho)+\gamma D_{x}^{2} \rho,
\end{array}\right.
$$

where $\boldsymbol{D}(\boldsymbol{v})=\left(\nabla \boldsymbol{v}+\nabla \boldsymbol{v}^{T}\right) / 2$ is the strain tensor and $D_{x}^{2} \rho$ is the hessian matrix of the density $\rho$. Here, the pressure $p$ and the coefficients $\alpha, \beta, \gamma, \delta, \mu$ and $\nu$ are functions of $\rho$. In the viscous stress tensor $\boldsymbol{S}$, we define the coefficients $\nu$ and $\mu$ as in [4]

$$
\nu=-\left(\mu_{0}+\tilde{\lambda}\right), \quad \mu=\mu_{0} \rho,
$$

where $\mu_{0}$ and $\tilde{\lambda}$ are viscosities of the fluid with $\mu_{0} \geq 0$ and $3 \tilde{\lambda}+2 \mu_{0} \geq 0$ and $\tilde{\lambda}+\frac{2}{3} \mu_{0}$ is called the bulk viscosity. Therefore, the viscous stress tensor reads

$$
\boldsymbol{S}=-\left(\left(\mu_{0}+\tilde{\lambda}\right) \operatorname{div} \boldsymbol{v}+p\right) \boldsymbol{I}+2 \mu_{0} \rho \boldsymbol{D}(\boldsymbol{v})
$$

and it follows that

$$
\operatorname{div} \boldsymbol{S}=-\left(\mu_{0}+\tilde{\lambda}\right) \nabla(\operatorname{div} \boldsymbol{v})-\nabla p+2 \mu_{0} \operatorname{div}(\rho \boldsymbol{D}(\boldsymbol{v})) .
$$

For the Korteweg stress tensor $\boldsymbol{K}$, we consider the following relations:

$$
\alpha=\kappa \rho, \quad \beta=\frac{\kappa}{2}, \quad \delta=-\kappa, \quad \gamma=0,
$$

for some constant $\kappa>0$. This choice corresponds precisely to the Korteweg's original assumptions connected with the variational theory of Van der Waals. In this case, the Korteweg stress tensor reads

$$
\boldsymbol{K}=\frac{\kappa}{2}\left(\Delta \rho^{2}-|\nabla \rho|^{2}\right) \boldsymbol{I}-\kappa(\nabla \rho \otimes \nabla \rho),
$$


and we obtain

$$
\operatorname{div} \boldsymbol{K}=\kappa \rho \nabla(\Delta \rho) .
$$

On the other hand, the non-solenoidal velocity effects and the Korteweg stresses arise in process of slow diffusion on miscible incompressible fluids, for example water and glycerin. In the physical experiment given by Joseph [10], the presence of sharp interfaces resembles familiar shapes which can be seen in immiscible liquids. The two fluids are characterized by their reference mass density: we denote by $\bar{\rho}_{1}$ the density of the first specie and $\bar{\rho}_{2}$ the density of the second one. We also need the velocity field of each constituent: $\boldsymbol{v}_{1}(t, \boldsymbol{x})$ and $\boldsymbol{v}_{2}(t, \boldsymbol{x})$, respectively. We define the volume fraction of the first specie:

$$
\phi(t, \boldsymbol{x})=\lim _{r \rightarrow 0} \frac{\text { Volume occupied at time } t \text { by the first specie in } B(\boldsymbol{x}, r)}{|B(\boldsymbol{x}, r)|},
$$

with $0 \leq \phi(t, \boldsymbol{x}) \leq 1$. Therefore, assuming that each fluid is incompressible and keeps a constant mass density, the density of the mixture is defined by

$$
\rho(t, \boldsymbol{x})=\underbrace{\bar{\rho}_{2}(1-\phi(t, \boldsymbol{x}))}_{:=\rho_{2}(t, \boldsymbol{x})}+\underbrace{\bar{\rho}_{1} \phi(t, \boldsymbol{x})}_{:=\rho_{1}(t, \boldsymbol{x})}=\bar{\rho}_{2}+\left(\bar{\rho}_{1}-\bar{\rho}_{2}\right) \phi(t, \boldsymbol{x}) .
$$

Writing the mass conservation for the two species, we obtain

$$
\partial_{t} \rho+\operatorname{div}(\rho \boldsymbol{v})=0
$$

where

$$
\rho \boldsymbol{v}(t, \boldsymbol{x})=\left(\rho_{2} \boldsymbol{v}_{2}+\rho_{1} \boldsymbol{v}_{1}\right)(t, \boldsymbol{x})
$$

defines the mean mass velocity (or barycentric velocity) $\boldsymbol{v}(t, \boldsymbol{x})$, which is not divergence free. By contrast, we define the mean volume velocity

$$
\boldsymbol{u}(t, \boldsymbol{x})=(1-\phi(t, \boldsymbol{x})) \boldsymbol{v}_{2}(t, \boldsymbol{x})+\phi(t, \boldsymbol{x}) \boldsymbol{v}_{1}(t, \boldsymbol{x}) .
$$

Using the definitions, we easily verify that the velocity field $\boldsymbol{u}$ is solenoidal.

According to Kazhikhov and Smagulov [14] (see also [1, 2]) we consider the following non-standard constraint associated to the pressure $p$ :

$$
\operatorname{div} \boldsymbol{v}=-\operatorname{div}(\lambda \nabla \ln (\rho)),
$$

where $\lambda>0$ is interpreted as a diffusion coefficient. This Fick's law describes the diffusive fluxes of one fluid into the other. Clearly, when we set

$$
\boldsymbol{v}=\boldsymbol{u}-\lambda \nabla \ln (\rho),
$$

the relation yields (12).

Let us prove that if we use the relation (13), then system (6) completed by (9) and (11) can be rewritten as the KSK model (1). More precisely, let us prove the following:

Lemma 2.1 Using the relation (13) with $\operatorname{div} \boldsymbol{u}=0$ and $\lambda=2 \mu_{0}$, then system (6) may be rewritten as system (1), with the modified pressure

$$
P=p-\frac{\lambda}{2}(\lambda+2 \tilde{\lambda}) \Delta \ln (\rho)-\kappa \rho \Delta \rho
$$


Proof. We use (13) into equations involving $\rho$ and $\boldsymbol{v}$, in order to eliminate $\boldsymbol{v}$. Clearly, the mixture density $\rho$ satisfies the mass conservation and we obtain

$$
\partial_{t} \rho+\operatorname{div}(\rho \boldsymbol{u})=\operatorname{div}(\lambda \nabla \rho) .
$$

For the momentum equation, we develop each term of $(6)_{2}$ taking into account (9) and (11). We start by using $(6)_{1}$ in order to write

$$
\partial_{t}(\rho \boldsymbol{v})+\operatorname{div}(\rho \boldsymbol{v} \otimes \boldsymbol{v})=\rho\left(\partial_{t} \boldsymbol{v}+(\boldsymbol{v} \cdot \nabla) \boldsymbol{v}\right)
$$

Then, using (13) we have

$\rho\left(\partial_{t} \boldsymbol{v}+(\boldsymbol{v} \cdot \nabla) \boldsymbol{v}\right)=\rho\left(\partial_{t} \boldsymbol{u}+(\boldsymbol{u} \cdot \nabla) \boldsymbol{u}\right)-\lambda \rho \nabla\left(\frac{\partial_{t} \rho}{\rho}\right)-\lambda \rho(\boldsymbol{u} \cdot \nabla) \nabla \ln \rho-\lambda(\nabla \rho \cdot \nabla) \boldsymbol{u}+\lambda^{2}(\nabla \rho \cdot \nabla) \nabla \ln \rho$, with

$$
\begin{aligned}
\rho \nabla\left(\frac{\partial_{t} \rho}{\rho}\right) & =\lambda \nabla \Delta \rho-\nabla(\boldsymbol{u} \cdot \nabla \rho)-\lambda \frac{\Delta \rho}{\rho} \nabla \rho+(\boldsymbol{u} \cdot \nabla \rho) \frac{\nabla \rho}{\rho}, \\
\rho(\boldsymbol{u} \cdot \nabla) \nabla \ln \rho & =(\boldsymbol{u} \cdot \nabla) \nabla \rho-(\boldsymbol{u} \cdot \nabla \rho) \frac{\nabla \rho}{\rho}, \\
(\nabla \rho \cdot \nabla) \nabla \ln \rho & =\frac{(\nabla \rho \cdot \nabla) \nabla \rho}{\rho}-\frac{|\nabla \rho|^{2}}{\rho^{2}} \nabla \rho .
\end{aligned}
$$

Also, taking into account that

$$
(\boldsymbol{u} \cdot \nabla) \nabla \rho-\nabla(\boldsymbol{u} \cdot \nabla \rho)=-\operatorname{div}\left(\rho \nabla \boldsymbol{u}^{T}\right)
$$

we obtain

$$
\begin{aligned}
\partial_{t}(\rho \boldsymbol{v})+\operatorname{div}(\rho \boldsymbol{v} \otimes \boldsymbol{v}) & =\rho\left(\partial_{t} \boldsymbol{u}+(\boldsymbol{u} \cdot \nabla) \boldsymbol{u}\right)-\lambda(\nabla \rho \cdot \nabla) \boldsymbol{u}+\lambda \operatorname{div}\left(\rho \nabla \boldsymbol{u}^{T}\right) \\
& +\lambda^{2}\left[\frac{\Delta \rho}{\rho} \nabla \rho+\frac{(\nabla \rho \cdot \nabla) \nabla \rho}{\rho}-\nabla \Delta \rho-\frac{|\nabla \rho|^{2}}{\rho^{2}} \nabla \rho\right] .
\end{aligned}
$$

Furthermore, using (12) and (13), each term of $\operatorname{div} \boldsymbol{S}$ reads

$$
\begin{aligned}
\left(\mu_{0}+\tilde{\lambda}\right) \nabla(\operatorname{div} \boldsymbol{v}) & =-\lambda\left(\mu_{0}+\tilde{\lambda}\right) \nabla \Delta \ln (\rho), \\
\mu_{0} \operatorname{div}(\rho \nabla \boldsymbol{v}) & =\mu_{0} \operatorname{div}(\rho \nabla \boldsymbol{u})+\mu_{0} \lambda\left[\frac{\Delta \rho}{\rho} \nabla \rho+\frac{(\nabla \rho \cdot \nabla) \nabla \rho}{\rho}-\nabla \Delta \rho-\frac{|\nabla \rho|^{2}}{\rho^{2}} \nabla \rho\right], \\
\mu_{0} \operatorname{div}\left(\rho \nabla \boldsymbol{v}^{T}\right) & =\mu_{0} \operatorname{div}\left(\rho \nabla \boldsymbol{u}^{T}\right)+\mu_{0} \lambda\left[\frac{\Delta \rho}{\rho} \nabla \rho+\frac{(\nabla \rho \cdot \nabla) \nabla \rho}{\rho}-\nabla \Delta \rho-\frac{|\nabla \rho|^{2}}{\rho^{2}} \nabla \rho\right] .
\end{aligned}
$$

Finally, by gathering all these relations and (11) together, the momentum equation $(6)_{2}$ is rewritten as:

$$
\begin{aligned}
\rho\left(\partial_{t} \boldsymbol{u}+(\boldsymbol{u} \cdot \nabla) \boldsymbol{u}\right) & -\lambda(\nabla \rho \cdot \nabla) \boldsymbol{u}+\lambda \operatorname{div}\left(\rho \nabla \boldsymbol{u}^{T}\right)-2 \mu_{0} \operatorname{div}(\rho \boldsymbol{D}(\boldsymbol{u}))-\lambda\left(\mu_{0}+\tilde{\lambda}\right) \nabla \Delta \ln (\rho)+\nabla p \\
+ & \underbrace{\left(\lambda^{2}-2 \mu_{0} \lambda\right)\left[\frac{\Delta \rho}{\rho} \nabla \rho+\frac{(\nabla \rho \cdot \nabla) \nabla \rho}{\rho}-\nabla \Delta \rho-\frac{|\nabla \rho|^{2}}{\rho^{2}} \nabla \rho\right]}_{(*)}=\rho \boldsymbol{g}+\kappa \rho \nabla(\Delta \rho) .
\end{aligned}
$$

However, as in [4], equation (15) can be simplified if we make the special choice $\lambda=2 \mu_{0}$. For this choice, the $\mathcal{O}\left(\lambda^{2}\right)$ terms marked with $\left(^{*}\right)$ in (15) disappear. Moreover, we observe that

$$
\kappa \rho \nabla(\Delta \rho)=\kappa \nabla(\rho \Delta \rho)-\kappa \nabla \rho \Delta \rho .
$$

In conclusion, equation $(1)_{1}$ is obtained by including all the gradient terms of $(15)$ in the modified pressure $P$. 
Remark 2.2 The model (1), without the Korteweg stress tensor (i.e. for $\kappa=0$ ), is called the pollutant model (see [4]). In particular, (1) is obtained without any assumption on the diffusivity $\lambda$.

Remark 2.3 In $[9,10,11]$ the solenoidal velocity $\boldsymbol{u}$ is related to $\boldsymbol{v}$ and the gradient of volume fraction $\phi$ by setting $\boldsymbol{v}=\boldsymbol{u}-\bar{\lambda}(\phi) \nabla \phi$, with a diffusion coefficient $\bar{\lambda}(\phi)>0$. This relation is justified by considering that the total mass of solute plus solvent is conserved for each volume $\Omega$, but solute and solvent diffuse in and out of $\Omega$. Consequently, the volume fraction $\phi$ of the solute satisfies

$$
\partial_{t} \phi+\operatorname{div}(\phi \boldsymbol{v})=\operatorname{div}(\bar{\lambda}(\phi) \nabla \phi) .
$$

The equivalence between (14) and (16) can be seen in [6].

\section{Functional setup}

Let us introduce the following functional spaces (see for instance [15], [18] for their properties):

$$
\begin{aligned}
\mathcal{V} & =\left\{\boldsymbol{u} \in \mathcal{D}(\Omega)^{3}: \operatorname{div} \boldsymbol{u}=0 \text { in } \Omega\right\} \\
\mathbf{V} & =\left\{\boldsymbol{u} \in \mathbf{H}_{0}^{1}(\Omega): \operatorname{div} \boldsymbol{u}=0 \text { in } \Omega\right\} \\
\mathbf{H} & =\left\{\boldsymbol{u} \in \mathbf{L}^{2}(\Omega): \operatorname{div} \boldsymbol{u}=0 \text { in } \Omega, \boldsymbol{u} \cdot \boldsymbol{n}=0 \text { on } \Gamma\right\} \\
H_{N}^{s} & =\left\{\rho \in H^{s}(\Omega): \frac{\partial \rho}{\partial \boldsymbol{n}}=0 \text { on } \Gamma, \int_{\Omega} \rho(\boldsymbol{x}) d \boldsymbol{x}=\int_{\Omega} \rho_{0}(\boldsymbol{x}) d \boldsymbol{x}\right\}, \quad s \geq 2 .
\end{aligned}
$$

$\mathbf{V}$ and $\mathbf{H}$ are the closures of $\mathcal{V}$ in $\mathbf{H}_{0}^{1}(\Omega)$ and $\mathbf{L}^{2}(\Omega)$ respectively.

Let us recall the definition of a weak solution for the KSK model (1). Such class of solutions can be found in [4] for the Kazhikhov-Smagulov type models and in [18] for the incompressible Navier-Stokes equations. Another equivalent weak formulation can be found in [1].

Definition 3.1 A pair of functions $(\boldsymbol{u}, \rho)$ is called a weak solution of problem (1),(2),(3) on $\Omega$ if and only if the following assumptions are satisfied:

1. $\boldsymbol{u} \in L^{\infty}(0, T ; \boldsymbol{H}) \cap L^{2}(0, T ; \boldsymbol{V})$,

$\rho \in L^{\infty}\left(0, T ; H^{1}(\Omega)\right) \cap L^{2}\left(0, T ; H_{N}^{2}\right)$,

$0<m \leq \rho(t, \boldsymbol{x}) \leq M<+\infty$, a.e. $(t, \boldsymbol{x}) \in \mathcal{Q}_{T}$.

2. For all $\phi \in \mathcal{C}^{1}([0, T] ; \boldsymbol{V})$ such that $\phi(T,)=$.0 , one has:

$$
\begin{aligned}
& \int_{0}^{T}\left\{-\left(\boldsymbol{u}, \rho \partial_{t} \boldsymbol{\phi}+((\rho \boldsymbol{u}-\lambda \nabla \rho) \cdot \nabla) \boldsymbol{\phi}\right)+\frac{\lambda}{2}\left(\rho\left(\nabla \boldsymbol{u}-\nabla \boldsymbol{u}^{T}\right), \nabla \boldsymbol{\phi}\right)\right\} d t \\
& =\int_{0}^{T}(\rho \boldsymbol{g}-\kappa \Delta \rho \nabla \rho, \boldsymbol{\phi}) d t+\left(\rho_{0} \boldsymbol{u}_{0}, \boldsymbol{\phi}(0)\right) .
\end{aligned}
$$

3. For all $\varphi \in \mathcal{C}^{1}\left([0, T] ; H^{1}(\Omega)\right)$ such that $\varphi(T,)=$.0 , one has:

$$
\int_{0}^{T}\left\{(\boldsymbol{u} \cdot \nabla \rho, \varphi)+\lambda(\nabla \rho, \nabla \varphi)-\left(\rho, \partial_{t} \varphi\right)\right\} d t=\left(\rho_{0}, \varphi(0)\right)
$$

Remark 3.2 The pressure $P$ associated with the weak solution $(\boldsymbol{u}, \rho)$ can be obtained using (17) and the Rham's lemma [18]. 


\section{Preliminary results}

In this section we recall some useful results concerning the convection-diffusion equation satisfied by the density function. This kind of results was used in the framework of compressible Navier-Stokes equations by Feireisl et al. [8] and also by Bresch et al. [4] for the Kazhikhov-Smagulov model. Given the initial density $\rho_{0}$ and the velocity field $\boldsymbol{u}$, we consider the density $\rho$ as solution of the following Neumann problem:

$$
\begin{cases}\partial_{t} \rho+\boldsymbol{u} \cdot \nabla \rho=\lambda \Delta \rho & \text { in } \mathcal{Q}_{T}, \\ \rho(0, \boldsymbol{x})=\rho_{0}(\boldsymbol{x}) & \text { in } \Omega, \\ \frac{\partial \rho}{\partial \boldsymbol{n}}=0 & \text { on } \Sigma .\end{cases}
$$

The density $\rho$ satisfies the maximum principle. This result is classical and may be found in [4].

Proposition 4.1 If $(\boldsymbol{u}, \rho)$ is a weak solution of (1), then

$$
0<m \leq \rho(t, \boldsymbol{x}) \leq M<+\infty \quad \text { a.e. }(t, \boldsymbol{x}) \in \mathcal{Q}_{T} .
$$

Proposition 4.2 Let $\rho_{0} \in H^{1}(\Omega)$ satisfy (4) and $\boldsymbol{u} \in \mathcal{C}\left([0, T] ; \boldsymbol{V} \cap \boldsymbol{H}^{2}(\Omega)\right)$. Then there exists a unique solution of (19) such that

$$
\rho \in L^{\infty}\left(0, T ; H^{1}(\Omega)\right) \cap L^{2}\left(0, T ; H_{N}^{2}\right) .
$$

Moreover, we have

$$
\begin{gathered}
\sup _{0 \leq t \leq T}\|\rho(t)\|_{L^{2}(\Omega)}^{2} \leq\left\|\rho_{0}\right\|_{H^{1}(\Omega)}^{2}, \\
\int_{0}^{T}\|\nabla \rho(t)\|_{L^{2}(\Omega)}^{2} d t \leq \frac{1}{2 \lambda}\left\|\rho_{0}\right\|_{H^{1}(\Omega)}^{2}, \\
\sup _{0 \leq t \leq T}\|\nabla \rho(t)\|_{L^{2}(\Omega)}^{2} \leq C_{\lambda}\left\|\rho_{0}\right\|_{H^{1}(\Omega)}^{2}\left(1+\sup _{0 \leq t \leq T}\|\boldsymbol{u}(t)\|_{L^{\infty}(\Omega)}^{2}\right), \\
\int_{0}^{T}\|\Delta \rho(t)\|_{L^{2}(\Omega)}^{2} d t \leq \frac{C_{\lambda}}{\lambda}\left\|\rho_{0}\right\|_{H^{1}(\Omega)}^{2}\left(1+\sup _{0 \leq t \leq T}\|\boldsymbol{u}(t)\|_{L^{\infty}(\Omega)}^{2}\right),
\end{gathered}
$$

where $C_{\lambda}$ is a positive constant depending only on $\lambda$.

For a given $\rho_{0} \in H^{1}(\Omega)$ which satisfies (4) and $\boldsymbol{u} \in \mathcal{C}\left([0, T] ; \mathbf{V} \cap \mathbf{H}^{2}(\Omega)\right)$, let $\rho$ the solution obtained by Proposition 4.2. Hence, it is clear that the following map

$$
\mathcal{S}: \mathcal{C}\left([0, T] ; \mathbf{V} \cap \mathbf{H}^{2}(\Omega)\right) \longrightarrow L^{\infty}\left(0, T ; H^{1}(\Omega)\right) \cap L^{2}\left(0, T ; H_{N}^{2}\right),
$$

such that $\rho=\mathcal{S} \boldsymbol{u}$, is well defined.

Proposition 4.3 Let $\rho_{0} \in H^{1}(\Omega)$ satisfy (4) and $\boldsymbol{u}_{1}, \boldsymbol{u}_{2} \in \mathcal{C}\left([0, T] ; \boldsymbol{V} \cap \boldsymbol{H}^{2}(\Omega)\right)$. Set $\rho=\rho_{1}-\rho_{2}=$ $\mathcal{S} \boldsymbol{u}_{1}-\mathcal{S} \boldsymbol{u}_{2}$ and $\boldsymbol{u}=\boldsymbol{u}_{1}-\boldsymbol{u}_{2}$, we have the following estimates:

$$
\sup _{0 \leq t \leq T}\|\rho(t)\|_{L^{2}(\Omega)}^{2}+\lambda \int_{0}^{T}\|\nabla \rho(t)\|_{L^{2}(\Omega)}^{2} d t \leq \frac{M^{2}}{\lambda} T \sup _{0 \leq t \leq T}\|\boldsymbol{u}(t)\|_{L^{2}(\Omega)}^{2},
$$




$$
\begin{aligned}
\sup _{0 \leq t \leq T}\|\nabla \rho(t)\|_{L^{2}(\Omega)}^{2}+\lambda \int_{0}^{T}\|\Delta \rho(t)\|_{L^{2}(\Omega)}^{2} d t & \leq \frac{2}{\lambda} T \sup _{0 \leq t \leq T}\left\|\nabla \rho_{1}(t)\right\|_{L^{2}(\Omega)}^{2} \sup _{0 \leq t \leq T}\|\boldsymbol{u}(t)\|_{L^{\infty}(\Omega)}^{2} \\
& +\frac{2 M^{2}}{\lambda^{3}} T \sup _{0 \leq t \leq T}\left\|\boldsymbol{u}_{2}(t)\right\|_{L^{\infty}(\Omega)}^{2} \sup _{0 \leq t \leq T}\|\boldsymbol{u}(t)\|_{L^{2}(\Omega)}^{2}
\end{aligned}
$$

Also, we recall that there exists an orthonormal basis of $\mathbf{L}^{2}(\Omega)$ defined by

$$
\begin{gathered}
\boldsymbol{\omega}_{k} \in \mathbf{V} \cap \mathbf{H}^{2}(\Omega) \\
-\mathbb{P} \boldsymbol{\Delta} \boldsymbol{\omega}_{k}=\lambda_{k} \boldsymbol{\omega}_{k} \quad \text { on } \Omega,
\end{gathered}
$$

where $\mathbb{P}$ is the orthogonal projection operator of $\mathbf{L}^{2}(\Omega)$ onto $\mathbf{H}$. For any $n \in \mathbb{N}^{*}$, we denote by $\mathbf{X}_{\mathbf{n}}$ the finite dimensional subspace of $\mathbf{H}$ such that

$$
\mathbf{X}_{\mathbf{n}}=\mathcal{V} \text { ect }\left\{\boldsymbol{\omega}_{k}, k=1, \ldots, n\right\}
$$

and we consider the orthogonal projection $\mathbb{P}_{n}: \mathbf{L}^{2}(\Omega) \rightarrow \mathbf{X}_{\mathbf{n}}$ defined by

$$
\forall \boldsymbol{w} \in \mathbf{H}, \quad\left(\mathbb{P}_{n} \boldsymbol{w}, \boldsymbol{v}\right)=(\boldsymbol{w}, \boldsymbol{v}), \quad \forall \boldsymbol{v} \in \mathbf{X}_{\mathbf{n}} .
$$

The operator $\mathbb{P}_{n}$ satisfies the following property:

Proposition $4.4 \mathbb{P}_{n}$ is continuous on $\boldsymbol{H}$ and it can be extended as a continuous operator on $\boldsymbol{V}$ and $\boldsymbol{V}^{\prime}$.

Following Feireisl et al. [8], we introduce a family of operators $\mathcal{M}[\rho]: \mathbf{X}_{\mathbf{n}} \longrightarrow \mathbf{X}_{\mathbf{n}}$ defined by

$$
(\mathcal{M}[\rho] \boldsymbol{v}, \boldsymbol{\omega})=\int_{\Omega} \rho \boldsymbol{v} \cdot \boldsymbol{\omega} d \boldsymbol{x} \quad \text { for all } \boldsymbol{v}, \boldsymbol{\omega} \in \mathbf{X}_{\mathbf{n}}
$$

It is clear that if $\rho \in L^{\infty}(\Omega)$, then $\mathcal{M}[\rho]$ is well defined. Moreover, assumed $m>0$, we set

$$
\mathcal{D}=\left\{\rho \in L^{\infty}(\Omega) ; \quad \rho(\boldsymbol{x}) \geq m>0\right\} .
$$

Proposition $4.5 \mathcal{M}[\rho]$ is one-to-one and its inverse satisfies

$$
\begin{aligned}
\left\|\mathcal{M}[\rho]^{-1}\right\|_{\mathcal{L}\left(\mathbf{X}_{\mathbf{n}}, \mathbf{X}_{\mathbf{n}}\right)} \leq\left(\inf _{\boldsymbol{x} \in \Omega} \rho(\boldsymbol{x})\right)^{-1} & \forall \rho \in \mathcal{D}, \\
\left\|\mathcal{M}\left[\rho_{1}\right]^{-1}-\mathcal{M}\left[\rho_{2}\right]^{-1}\right\|_{\mathcal{L}\left(\mathbf{X}_{\mathbf{n}}, \mathbf{X}_{\mathbf{n}}\right)} \leq \frac{C_{n}}{m^{2}}\left\|\rho_{1}-\rho_{2}\right\|_{L^{2}(\Omega)} & \forall \rho_{1}, \rho_{2} \in \mathcal{D},
\end{aligned}
$$

where $C_{n}$ is a constant depending on the dimension of $\mathbf{X}_{\mathbf{n}}$.

\section{$5 \quad$ Proof of Theorem 1.1}

\subsection{The Faedo-Galerkin method}

The approximate solutions

$$
\left(\boldsymbol{u}_{n}, \rho_{n}\right) \in \mathcal{C}\left([0, T] ; \mathbf{X}_{\mathbf{n}}\right) \times \mathcal{C}\left([0, T] ; H^{1}(\Omega) \cap H_{N}^{2}\right)
$$


we shall look for are required to satisfy

$$
\begin{cases}\int_{\Omega} \partial_{t}\left(\rho_{n} \boldsymbol{u}_{n}\right) \cdot \boldsymbol{v} d \boldsymbol{x}+\int_{\Omega}\left(\boldsymbol{u}_{n} \cdot \nabla \rho_{n}\right) \boldsymbol{u}_{n} \cdot \boldsymbol{v} d \boldsymbol{x}-\lambda \int_{\Omega} \Delta \rho_{n} \boldsymbol{u}_{n} \cdot \boldsymbol{v} d \boldsymbol{x}+\int_{\Omega}\left(\left(\rho_{n} \boldsymbol{u}_{n}-\lambda \nabla \rho_{n}\right) \cdot \nabla\right) \boldsymbol{u}_{n} \cdot \boldsymbol{v} d \boldsymbol{x} \\ -\frac{\lambda}{2} \int_{\Omega} \operatorname{div}\left(\rho_{n} \nabla \boldsymbol{u}_{n}-\rho_{n} \nabla \boldsymbol{u}_{n}^{T}\right) \cdot \boldsymbol{v} d \boldsymbol{x}=\int_{\Omega} \rho_{n} \boldsymbol{g} \cdot \boldsymbol{v} d \boldsymbol{x}-\kappa \int_{\Omega} \Delta \rho_{n} \nabla \rho_{n} \cdot \boldsymbol{v} d \boldsymbol{x}, & \forall \boldsymbol{v} \in \mathbf{X}_{\mathbf{n}}, \\ \int_{\Omega} \partial_{t}\left(\rho_{n}\right) \eta d \boldsymbol{x}+\int_{\Omega} \boldsymbol{u}_{n} \cdot \nabla \rho_{n} \eta d \boldsymbol{x}=\lambda \int_{\Omega} \Delta \rho_{n} \eta d \boldsymbol{x}, & \\ \boldsymbol{u}_{n}(0)=\boldsymbol{u}_{0 n}=\mathbb{P}_{n} \boldsymbol{u}_{0}, & \\ \rho_{n}(0)=\rho_{0} . & \end{cases}
$$

We set

$\mathcal{N}\left[\boldsymbol{u}_{n}, \rho_{n}\right]=-\left(\left(\rho_{n} \boldsymbol{u}_{n}-\lambda \nabla \rho_{n}\right) \cdot \nabla\right) \boldsymbol{u}_{n}+\frac{\lambda}{2} \operatorname{div}\left(\rho_{n} \nabla \boldsymbol{u}_{n}-\rho_{n} \nabla \boldsymbol{u}_{n}^{T}\right)-\left(\boldsymbol{u}_{n} \cdot \nabla \rho_{n}\right) \boldsymbol{u}_{n}+\lambda \Delta \rho_{n} \boldsymbol{u}_{n}-\kappa \Delta \rho_{n} \nabla \rho_{n}+\rho_{n} \boldsymbol{g}$.

Taking (31) 1 with $\boldsymbol{v}=\boldsymbol{\omega}_{k}$, for $k=1, \ldots, n$, and integrating in time between 0 and $t \leq T$, the solution $\boldsymbol{u}_{n}$ satisfies the following integral equations for $k=1, \ldots, n$ :

$$
\int_{\Omega} \rho_{n}(t) \boldsymbol{u}_{n}(t) \cdot \boldsymbol{\omega}_{k} d \boldsymbol{x}=\int_{\Omega} \boldsymbol{q}_{0} \cdot \boldsymbol{\omega}_{k} d \boldsymbol{x}+\int_{0}^{t} \int_{\Omega} \mathcal{N}\left[\boldsymbol{u}_{n}, \rho_{n}\right] \cdot \boldsymbol{\omega}_{k} d \boldsymbol{x} d s
$$

where $\rho_{n}=\mathcal{S} \boldsymbol{u}_{n}$ and $\boldsymbol{q}_{0}=\rho_{0} \boldsymbol{u}_{0 n}$. Using (27) and (28), we rewrite (33) as follows:

$$
\left(\mathcal{M}\left[\rho_{n}(t)\right] \boldsymbol{u}_{n}(t), \boldsymbol{\omega}_{k}\right)=\left(\mathbb{P}_{n} \boldsymbol{q}_{0}, \boldsymbol{\omega}_{k}\right)+\left(\mathbb{P}_{n} \int_{0}^{t} \mathcal{N}\left[\boldsymbol{u}_{n}(s), \rho_{n}(s)\right] d s, \boldsymbol{\omega}_{k}\right),
$$

for $k=1, \ldots, n$. Therefore, for all $t \in[0, T]$, we have

$$
\mathcal{M}\left[\rho_{n}(t)\right] \boldsymbol{u}_{n}(t)=\mathbb{P}_{n} \boldsymbol{q}_{0}+\mathbb{P}_{n}\left(\int_{0}^{t} \mathcal{N}\left[\boldsymbol{u}_{n}(s), \rho_{n}(s)\right] d s\right) .
$$

Since $\mathcal{M}\left[\rho_{n}\right]$ is invertible, then the resulting equation reads

$$
\boldsymbol{u}_{n} \in \mathcal{C}\left([0, T] ; \mathbf{X}_{\mathbf{n}}\right), \quad \boldsymbol{u}_{n}(t)=\mathcal{M}\left[\rho_{n}(t)\right]^{-1} \mathbb{P}_{n}\left(\boldsymbol{q}_{0}+\int_{0}^{t} \mathcal{N}\left[\boldsymbol{u}_{n}(s), \rho_{n}(s)\right] d s\right)
$$

At this stage, $\boldsymbol{u}_{n}$ appears as a fixed point of a suitable functional $\Psi$

$$
\begin{aligned}
\Psi: \mathcal{C}\left([0, T] ; \mathbf{X}_{\mathbf{n}}\right) & \longrightarrow \mathcal{C}\left([0, T] ; \mathbf{X}_{\mathbf{n}}\right) \\
\boldsymbol{u}_{n} & \longmapsto \Psi\left(\boldsymbol{u}_{n}\right)
\end{aligned}
$$

defined by

$$
\Psi\left(\boldsymbol{u}_{n}\right)(t)=\mathcal{M}\left[\rho_{n}(t)\right]^{-1} \mathbb{P}_{n}\left(\boldsymbol{q}_{0}+\int_{0}^{t} \mathcal{N}\left[\boldsymbol{u}_{n}(s), \rho_{n}(s)\right] d s\right), \quad \text { for all } t \in[0, T]
$$

Let $\mathbf{X}_{\mathbf{T}}$ be the Banach space $\mathcal{C}\left([0, T] ; \mathbf{X}_{\mathbf{n}}\right)$ endowed with the norm

$$
\left\|\boldsymbol{u}_{n}\right\|_{\mathbf{x}_{\mathbf{T}}}=\sup _{0 \leq t \leq T}\left\|\boldsymbol{u}_{n}(t)\right\|_{\mathbf{L}^{2}(\Omega)} .
$$

In order to apply the Banach fixed point theorem, we shall establish some uniform estimates for $\Psi$. In the sequel, $C$ is constant and it changes from one line to another and may depend on different parameters that will be precise later on. 
Proposition 5.1 There exists a constant $C>0$ depending on $n, \lambda, \kappa, M, m,\left\|\rho_{0}\right\|_{H^{1}(\Omega)},\|\boldsymbol{g}\|_{L^{2}\left(0, T ; L^{2}(\Omega)\right)}$, such that for all $\boldsymbol{u}_{n} \in \mathbf{X}_{\mathbf{T}}$,

$$
\left\|\Psi\left(\boldsymbol{u}_{n}\right)\right\|_{\mathbf{x}_{\mathbf{T}}} \leq \frac{M}{m}\left\|\boldsymbol{u}_{0}\right\|_{L^{2}(\Omega)}+C \max \left(T, T^{\frac{1}{4}}\right)\left[1+\left\|\boldsymbol{u}_{n}\right\|_{\mathbf{x}_{\mathbf{T}}}^{2}\right]
$$

and for all $\boldsymbol{u}_{n}^{1}, \boldsymbol{u}_{n}^{2} \in \mathbf{X}_{\mathbf{T}}$,

$$
\left\|\Psi\left(\boldsymbol{u}_{n}^{1}\right)-\Psi\left(\boldsymbol{u}_{n}^{2}\right)\right\|_{\mathbf{x}_{\mathbf{T}}} \leq C \max \left(T, T^{\frac{1}{4}}\right)\left[1+\left\|\boldsymbol{u}_{0}\right\|_{L^{2}(\Omega)}+\left\|\boldsymbol{u}_{n}^{1}\right\|_{\mathbf{x}_{\mathbf{T}}}^{2}+\left\|\boldsymbol{u}_{n}^{2}\right\|_{\mathbf{x}_{\mathbf{T}}}^{2}\right]\left\|\boldsymbol{u}_{n}^{1}-\boldsymbol{u}_{n}^{2}\right\|_{\mathbf{x}_{\mathbf{T}}} .
$$

Proof. We have for all $\boldsymbol{u}_{n} \in \mathbf{X}_{\mathbf{T}}$ and for $t \leq T$

$$
\left\|\Psi\left(\boldsymbol{u}_{n}\right)(t)\right\|_{L^{2}(\Omega)} \leq\left\|\mathcal{M}\left[\rho_{n}(t)\right]^{-1}\right\|_{\mathcal{L}\left(\mathbf{X}_{\mathbf{n}}, \mathbf{X}_{\mathbf{n}}\right)}\left\|\mathbb{P}_{n}\left(\boldsymbol{q}_{0}+\int_{0}^{t} \mathcal{N}\left[\boldsymbol{u}_{n}(s), \rho_{n}(s)\right] d s\right)\right\|_{L^{2}(\Omega)} .
$$

We will use several times that on a finite dimensional subspace all the norms are equivalent. For instance we have

$$
\left\|\mathbb{P}_{n}\left(\Delta \rho_{n} \nabla \rho_{n}\right)\right\|_{L^{2}(\Omega)} \leq C_{n}\left\|\mathbb{P}_{n}\left(\Delta \rho_{n} \nabla \rho_{n}\right)\right\|_{H^{-1}(\Omega)}
$$

Moreover, using the continuity of $\mathbb{P}_{n}$ in $\mathbf{V}^{\prime}$, the embedding $L^{4 / 3}(\Omega) \subset H^{-1}(\Omega)$, the Hölder inequality and the following inequality:

$$
\|\nabla \rho\|_{L^{4}(\Omega)} \leq C_{0}\|\rho\|_{L^{\infty}(\Omega)}^{1 / 2}\|\Delta \rho\|_{L^{2}(\Omega)}^{1 / 2},
$$

together with Proposition 4.1, we get

$$
\left\|\mathbb{P}_{n}\left(\Delta \rho_{n} \nabla \rho_{n}\right)\right\|_{L^{2}(\Omega)} \leq C_{n} \sqrt{M}\left\|\Delta \rho_{n}\right\|_{L^{2}(\Omega)}^{3 / 2} .
$$

Employing the same arguments for the other terms of (32), we obtain

$$
\begin{array}{r}
\left\|\mathbb{P}_{n}\left(\mathcal{N}\left[\boldsymbol{u}_{n}, \rho_{n}\right]\right)\right\|_{L^{2}(\Omega)} \leq C\left(\left\|\boldsymbol{u}_{n}\right\|_{L^{2}(\Omega)}^{2}+\left\|\boldsymbol{u}_{n}\right\|_{L^{2}(\Omega)}\left\|\nabla \rho_{n}\right\|_{L^{2}(\Omega)}+\left\|\boldsymbol{u}_{n}\right\|_{L^{2}(\Omega)}+\left\|\boldsymbol{u}_{n}\right\|_{L^{2}(\Omega)}\left\|\Delta \rho_{n}\right\|_{L^{2}(\Omega)}\right. \\
\left.+\left\|\Delta \rho_{n}\right\|_{L^{2}(\Omega)}^{3 / 2}+\left\|\boldsymbol{u}_{n}\right\|_{L^{2}(\Omega)}^{2}\left\|\nabla \rho_{n}\right\|_{L^{2}(\Omega)}+\|\boldsymbol{g}\|_{L^{2}(\Omega)}\right) .
\end{array}
$$

Using (29) in (37), we obtain for $t \leq T$

$$
\begin{array}{r}
\left\|\Psi\left(\boldsymbol{u}_{n}\right)(t)\right\|_{L^{2}(\Omega)} \leq \frac{M}{m}\left\|\boldsymbol{u}_{0}\right\|_{L^{2}(\Omega)}+ \\
+\frac{C}{m}\left(T\left\|\boldsymbol{u}_{n}\right\|_{\mathbf{x}_{\mathbf{T}}}^{2}+\left\|\boldsymbol{u}_{n}\right\|_{\mathbf{x}_{\mathbf{T}}} \int_{0}^{t}\left\|\nabla \rho_{n}\right\|_{L^{2}(\Omega)} d s\right. \\
+\left\|\boldsymbol{u}_{n}\right\|_{\mathbf{x}_{\mathbf{T}}}^{2} \int_{0}^{t}\left\|\nabla \rho_{n}\right\|_{L^{2}(\Omega)} d s+T\left\|\boldsymbol{u}_{n}\right\|_{\mathbf{x}_{\mathbf{T}}} \\
\left.+\left\|\boldsymbol{u}_{n}\right\|_{\mathbf{x}_{\mathbf{T}}} \int_{0}^{t}\left\|\Delta \rho_{n}\right\|_{L^{2}(\Omega)} d s+\int_{0}^{t}\left\|\Delta \rho_{n}\right\|_{L^{2}(\Omega)}^{3 / 2} d s+\int_{0}^{t}\|\boldsymbol{g}\|_{L^{2}(\Omega)} d s\right),
\end{array}
$$

and taking into account (22) and (24), we get

$$
\begin{array}{r}
\left\|\Psi\left(\boldsymbol{u}_{n}\right)\right\|_{\mathbf{x}_{\mathbf{T}}} \leq \frac{M}{m}\left\|\boldsymbol{u}_{0}\right\|_{L^{2}(\Omega)}+\frac{C}{m}\left(T\left\|\boldsymbol{u}_{n}\right\|_{\mathbf{x}_{\mathbf{T}}}^{2}+\frac{T^{\frac{1}{2}}}{\sqrt{2 \lambda}}\left\|\rho_{0}\right\|_{H^{1}(\Omega)}\left\|\boldsymbol{u}_{n}\right\|_{\mathbf{x}_{\mathbf{T}}}\right. \\
+\frac{C_{\lambda} T^{\frac{1}{2}}}{\sqrt{\lambda}}\left\|\rho_{0}\right\|_{H^{1}(\Omega)}\left\|\boldsymbol{u}_{n}\right\|_{\mathbf{x}_{\mathbf{T}}}\left(1+\left\|\boldsymbol{u}_{n}\right\|_{\mathbf{x}_{\mathbf{T}}}^{2}\right)^{1 / 2}+T\left\|\boldsymbol{u}_{n}\right\|_{\mathbf{x}_{\mathbf{T}}}+\frac{T^{\frac{1}{2}}}{\sqrt{2 \lambda}}\left\|\rho_{0}\right\|_{H^{1}(\Omega)}\left\|\boldsymbol{u}_{n}\right\|_{\mathbf{x}_{\mathbf{T}}}^{2} \\
\left.+T^{\frac{1}{4}}\left(\frac{C_{\lambda}}{\lambda}\left\|\rho_{0}\right\|_{H^{1}(\Omega)}^{2}\left(1+\left\|\boldsymbol{u}_{n}\right\|_{\mathbf{x}_{\mathbf{T}}}^{2}\right)\right)^{3 / 4}+T^{\frac{1}{2}}\|\boldsymbol{g}\|_{L^{2}\left(0, T ; L^{2}(\Omega)\right)}\right) .
\end{array}
$$


Hence, we have

$$
\begin{aligned}
\left\|\Psi\left(\boldsymbol{u}_{n}\right)\right\|_{\mathbf{x}_{\mathbf{T}}} & \leq \frac{M}{m}\left\|\boldsymbol{u}_{0}\right\|_{L^{2}(\Omega)}+C \max \left(T, T^{\frac{1}{4}}\right)\left(\left\|\boldsymbol{u}_{n}\right\|_{\mathbf{x}_{\mathbf{T}}}^{2}+\left\|\boldsymbol{u}_{n}\right\|_{\mathbf{x}_{\mathbf{T}}}\right. \\
& \left.+\left\|\boldsymbol{u}_{n}\right\|_{\mathbf{x}_{\mathbf{T}}}\left(1+\left\|\boldsymbol{u}_{n}\right\|_{\mathbf{x}_{\mathbf{T}}}^{2}\right)^{1 / 2}+\left(1+\left\|\boldsymbol{u}_{n}\right\|_{\mathbf{x}_{\mathbf{T}}}^{2}\right)^{3 / 4}+1\right)
\end{aligned}
$$

where $C$ depend on $n, \lambda, \kappa, M, m,\left\|\rho_{0}\right\|_{H^{1}(\Omega)}$ and $\|\boldsymbol{g}\|_{L^{2}\left(0, T ; L^{2}(\Omega)\right)}$. Using the Young inequality in (39), we get easily

$$
\left\|\Psi\left(\boldsymbol{u}_{n}\right)\right\|_{\mathbf{x}_{\mathbf{T}}} \leq \frac{M}{m}\left\|\boldsymbol{u}_{0}\right\|_{L^{2}(\Omega)}+C \max \left(T, T^{\frac{1}{4}}\right)\left[1+\left\|\boldsymbol{u}_{n}\right\|_{\mathbf{x}_{\mathbf{T}}}^{2}\right]
$$

In order to prove (36), let us consider $\boldsymbol{u}_{n}^{1}, \boldsymbol{u}_{n}^{2} \in \mathbf{X}_{\mathbf{T}}$ and we set $\rho_{n}^{1}=\mathcal{S} \boldsymbol{u}_{n}^{1}, \rho_{n}^{2}=\mathcal{S} \boldsymbol{u}_{n}^{2}$ the solutions obtained by Proposition 4.2. Then, we find

$$
\begin{aligned}
\left\|\Psi\left(\boldsymbol{u}_{n}^{1}\right)(t)-\Psi\left(\boldsymbol{u}_{n}^{2}\right)(t)\right\|_{L^{2}(\Omega)} & \leq\left\|\left(\mathcal{M}\left[\rho_{n}^{1}(t)\right]^{-1}-\mathcal{M}\left[\rho_{n}^{2}(t)\right]^{-1}\right)\left(\mathbb{P}_{n} \boldsymbol{q}_{0}\right)\right\|_{L^{2}(\Omega)} \\
& +\| \mathcal{M}\left[\rho_{n}^{1}(t)\right]^{-1}\left(\mathbb{P}_{n}\left(\int_{0}^{t} \mathcal{N}\left[\boldsymbol{u}_{n}^{1}(s), \rho_{n}^{1}(s)\right]\right)\right) \\
& -\mathcal{M}\left[\rho_{n}^{2}(t)\right]^{-1}\left(\mathbb{P}_{n}\left(\int_{0}^{t} \mathcal{N}\left[\boldsymbol{u}_{n}^{2}(s), \rho_{n}^{2}(s)\right]\right)\right) \|_{L^{2}(\Omega)} .
\end{aligned}
$$

Thanks to Propositions 4.3 and 4.5 , we obtain

$$
\begin{aligned}
\left\|\left(\mathcal{M}\left[\rho_{n}^{1}(t)\right]^{-1}-\mathcal{M}\left[\rho_{n}^{2}(t)\right]^{-1}\right)\left(\mathbb{P}_{n} \boldsymbol{q}_{0}\right)\right\|_{L^{2}(\Omega)} & \leq\left\|\mathcal{M}\left[\rho_{n}^{1}(t)\right]^{-1}-\mathcal{M}\left[\rho_{n}^{2}(t)\right]^{-1}\right\| \mathcal{L}_{\left(\mathbf{X}_{\mathbf{n}}, \mathbf{X}_{\mathbf{n}}\right)}\left\|\mathbb{P}_{n} \boldsymbol{q}_{0}\right\|_{L^{2}(\Omega)} \\
& \leq C_{n} \frac{M}{m^{2}}\left\|\boldsymbol{u}_{0}\right\|_{L^{2}(\Omega)}\left\|\rho_{n}^{1}(t)-\rho_{n}^{2}(t)\right\|_{L^{2}(\Omega)} \\
& \leq C_{n} \frac{M^{2}}{m^{2} \sqrt{\lambda}} T^{1 / 2}\left\|\boldsymbol{u}_{0}\right\|_{L^{2}(\Omega)}\left\|\boldsymbol{u}_{n}^{1}-\boldsymbol{u}_{n}^{2}\right\|_{\mathbf{x}_{\mathbf{T}}} .
\end{aligned}
$$

We rewrite the last two terms of (40) as follows:

$$
\begin{aligned}
& \mathcal{M}\left[\rho_{n}^{1}(t)\right]^{-1}\left(\mathbb{P}_{n}\left(\int_{0}^{t} \mathcal{N}\left[\boldsymbol{u}_{n}^{1}, \rho_{n}^{1}\right] d s\right)\right)-\mathcal{M}\left[\rho_{n}^{2}(t)\right]^{-1}\left(\mathbb{P}_{n}\left(\int_{0}^{t} \mathcal{N}\left[\boldsymbol{u}_{n}^{2}, \rho_{n}^{2}\right] d s\right)\right) \\
& =\left(\mathcal{M}\left[\rho_{n}^{1}(t)\right]^{-1}-\mathcal{M}\left[\rho_{n}^{2}(t)\right]^{-1}\right)\left(\mathbb{P}_{n}\left(\int_{0}^{t} \mathcal{N}\left[\boldsymbol{u}_{n}^{1}, \rho_{n}^{1}\right] d s\right)\right)+\mathcal{M}\left[\rho_{n}^{2}(t)\right]^{-1}\left(\mathbb{P}_{n}\left(\int_{0}^{t}\left(\mathcal{N}\left[\boldsymbol{u}_{n}^{1}, \rho_{n}^{1}\right]-\mathcal{N}\left[\boldsymbol{u}_{n}^{2}, \rho_{n}^{2}\right]\right) d s\right)\right) \\
& =K_{1}+K_{2}
\end{aligned}
$$

For the term $K_{1}$, Proposition 4.5 gives

$$
\begin{aligned}
& \left\|\left(\mathcal{M}\left[\rho_{n}^{1}(t)\right]^{-1}-\mathcal{M}\left[\rho_{n}^{2}(t)\right]^{-1}\right)\left(\mathbb{P}_{n}\left(\int_{0}^{t} \mathcal{N}\left[\boldsymbol{u}_{n}^{1}(s), \rho_{n}^{1}(s)\right] d s\right)\right)\right\|_{L^{2}(\Omega)} \\
& \quad \leq\left\|\mathcal{M}\left[\rho_{n}^{1}(t)\right]^{-1}-\mathcal{M}\left[\rho_{n}^{2}(t)\right]^{-1}\right\|_{\mathcal{L}\left(\mathbf{X}_{\mathbf{n}}, \mathbf{X}_{\mathbf{n}}\right)}\left\|\mathbb{P}_{n}\left(\int_{0}^{t} \mathcal{N}\left[\boldsymbol{u}_{n}^{1}(s), \rho_{n}^{1}(s)\right] d s\right)\right\|_{L^{2}(\Omega)} \\
& \quad \leq C \max \left(T, T^{\frac{1}{4}}\right)\left[1+\left\|\boldsymbol{u}_{n}^{1}\right\|_{\mathbf{x}_{\mathbf{T}}}^{2}\right]\left\|\boldsymbol{u}_{n}^{1}-\boldsymbol{u}_{n}^{2}\right\|_{\mathbf{x}_{\mathbf{T}}} .
\end{aligned}
$$

For the term $K_{2}$, we have

$$
\begin{aligned}
\| & \mathcal{M}\left[\rho_{n}^{2}(t)\right]^{-1}\left(\mathbb{P}_{n}\left(\int_{0}^{t}\left(\mathcal{N}\left[\boldsymbol{u}_{n}^{1}(s), \rho_{n}^{1}(s)\right]-\mathcal{N}\left[\boldsymbol{u}_{n}^{2}(s), \rho_{n}^{2}(s)\right]\right) d s\right)\right) \|_{L^{2}(\Omega)} \\
& \leq\left\|\mathcal{M}\left[\rho_{n}^{2}(t)\right]^{-1}\right\|\left\|_{\mathcal{L}\left(\mathbf{X}_{\mathbf{n}}, \mathbf{X}_{\mathbf{n}}\right)} \int_{0}^{T}\right\| \mathbb{P}_{n}\left(\mathcal{N}\left[\boldsymbol{u}_{n}^{1}(t), \rho_{n}^{1}(t)\right]-\mathcal{N}\left[\boldsymbol{u}_{n}^{2}(t), \rho_{n}^{2}(t)\right]\right) \|_{L^{2}(\Omega)} d t \\
& \leq \frac{1}{m} \int_{0}^{T}\left\|\mathbb{P}_{n}\left(\mathcal{N}\left[\boldsymbol{u}_{n}^{1}(t), \rho_{n}^{1}(t)\right]-\mathcal{N}\left[\boldsymbol{u}_{n}^{2}(t), \rho_{n}^{2}(t)\right]\right)\right\|_{L^{2}(\Omega)} d t .
\end{aligned}
$$


Then, several calculations similar to those employed in (41) give us

$\int_{0}^{T}\left\|\mathbb{P}_{n}\left(\mathcal{N}\left[\boldsymbol{u}_{n}^{1}(t), \rho_{n}^{1}(t)\right]-\mathcal{N}\left[\boldsymbol{u}_{n}^{2}(t), \rho_{n}^{2}(t)\right]\right)\right\|_{L^{2}(\Omega)} d t \leq C \max \left(T, T^{\frac{1}{4}}\right)\left[1+\left\|\boldsymbol{u}_{n}^{1}\right\|_{\mathbf{x}_{\mathbf{T}}}^{2}+\left\|\boldsymbol{u}_{n}^{2}\right\|_{\mathbf{x}_{\mathbf{T}}}^{2}\right]\left\|\boldsymbol{u}_{n}^{1}-\boldsymbol{u}_{n}^{2}\right\|_{\mathbf{x}_{\mathbf{T}}}$.

Finally, gathering all the preceding estimates together, we find (36).

From now on, we set $R=2 \frac{M}{m}\left\|\boldsymbol{u}_{0}\right\|_{L^{2}(\Omega)}$ and $\mathcal{B}_{R}^{T}=\left\{\boldsymbol{u} \in \mathbf{X}_{\mathbf{T}}, \quad\|\boldsymbol{u}\|_{\mathbf{x}_{\mathbf{T}}} \leq R\right\}$.

Proposition 5.2 There exists $\left.T_{n} \in\right] 0,1\left[\right.$ small enough and $\boldsymbol{u}_{n} \in \mathcal{B}_{R}^{T_{n}}$ such that

$$
\boldsymbol{u}_{n}=\Psi\left(\boldsymbol{u}_{n}\right)
$$

Proof. Let $0<T_{n}<1$ such that

$$
\max \left(C T_{n}^{\frac{1}{4}}\left[R+\frac{1}{R}\right], C T_{n}^{\frac{1}{4}}\left[1+\left\|\boldsymbol{u}_{0}\right\|_{L^{2}(\Omega)}+2 R^{2}\right]\right) \leq \frac{1}{2} .
$$

Using Proposition 5.1, we prove that $\Psi$ is a contraction mapping on $\mathcal{B}_{R}^{T_{n}}$ and we deduce the existence of a unique fixed point of $\Psi$.

It is clear that $\boldsymbol{u}_{n}$, the fixed point of $\Psi$ obtained in Proposition 5.2, yields that $\left(\boldsymbol{u}_{n}, \rho_{n}=\mathcal{S} \boldsymbol{u}_{n}\right)$ is a local solution of the approximate problem (31). Now, we will prove that this local solution is, in fact, a global one. For this purpose we establish uniform estimates for $\left(\boldsymbol{u}_{n}, \rho_{n}\right)$ with respect to time.

Proposition 5.3 There exists a constant $C>0$ depending on $\rho_{0}, \boldsymbol{u}_{0}, \boldsymbol{g}, M, m, \lambda, \kappa$, such that for all $t \in\left[0, T_{n}\right)$

$$
\begin{gathered}
m\left\|\boldsymbol{u}_{n}(t)\right\|_{L^{2}(\Omega)}^{2}+\frac{\lambda m}{4} \int_{0}^{t}\left\|\nabla \boldsymbol{u}_{n}(s)\right\|_{L^{2}(\Omega)}^{2} d s \leq C, \\
\kappa\left\|\nabla \rho_{n}(t)\right\|_{L^{2}(\Omega)}^{2}+2 \kappa \lambda \int_{0}^{t}\left\|\Delta \rho_{n}(s)\right\|_{L^{2}(\Omega)}^{2} d s \leq C .
\end{gathered}
$$

Proof. Firstly, taking $\boldsymbol{v}=\boldsymbol{u}_{n}(t)$ in $(31)_{1}$, we obtain

$$
\frac{1}{2} \frac{d}{d t} \int_{\Omega} \rho_{n}\left|\boldsymbol{u}_{n}\right|^{2} d \boldsymbol{x}+\frac{\lambda}{2} \int_{\Omega} \rho_{n}\left(\nabla \boldsymbol{u}_{n}-\nabla \boldsymbol{u}_{n}^{T}\right): \nabla \boldsymbol{u}_{n} d \boldsymbol{x}=\int_{\Omega} \rho_{n} \boldsymbol{g} \cdot \boldsymbol{u}_{n} d \boldsymbol{x}-\kappa \int_{\Omega} \Delta \rho_{n} \nabla \rho_{n} \cdot \boldsymbol{u}_{n} d \boldsymbol{x}
$$

Next, by $(31)_{2}$ we have

$$
\partial_{t} \rho_{n}-\lambda \Delta \rho_{n}=-\boldsymbol{u}_{n} \cdot \nabla \rho_{n}, \quad \text { in the distribution sens on } \mathcal{Q}_{T} .
$$

Since $\left(\boldsymbol{u}_{n}, \rho_{n}\right) \in \mathcal{C}\left(\left[0, T_{n}\right] ; \mathbf{X}_{\mathbf{n}}\right) \times L^{\infty}\left(0, T_{n} ; H^{1}(\Omega)\right) \cap L^{2}\left(0, T_{n} ; H_{N}^{2}\right)$, we conclude that

$$
\partial_{t} \rho_{n} \in L^{2}\left(0, T_{n} ; L^{2}(\Omega)\right)
$$

Now, we multiply (45) by $-\kappa \Delta \rho_{n}(t)$ and after integration by parts, we deduce that

$$
\frac{\kappa}{2} \frac{d}{d t}\left\|\nabla \rho_{n}\right\|_{L^{2}(\Omega)}^{2}+\kappa \lambda\left\|\Delta \rho_{n}\right\|_{L^{2}(\Omega)}^{2}=\kappa \int_{\Omega} \Delta \rho_{n} \boldsymbol{u}_{n} \cdot \nabla \rho_{n} d \boldsymbol{x} .
$$

By adding (44) and (46), we get

$$
\frac{d}{d t}\left(\left\|\sqrt{\rho_{n}} \boldsymbol{u}_{n}\right\|_{L^{2}(\Omega)}^{2}+\kappa\left\|\nabla \rho_{n}\right\|_{L^{2}(\Omega)}^{2}\right)+2 \kappa \lambda\left\|\Delta \rho_{n}\right\|_{L^{2}(\Omega)}^{2}+\lambda \int_{\Omega} \rho_{n}\left(\nabla \boldsymbol{u}_{n}-\nabla \boldsymbol{u}_{n}^{T}\right): \nabla \boldsymbol{u}_{n} d \boldsymbol{x}=2 \int_{\Omega} \rho_{n} \boldsymbol{g} \cdot \boldsymbol{u}_{n} d \boldsymbol{x} .
$$


Using the so-called vorticity tensor $\mathbf{W}=\nabla \boldsymbol{u}_{n}-\nabla \boldsymbol{u}_{n}^{T}$ (see [4]), and observing that

$$
\int_{\Omega} \rho_{n}\left(\nabla \boldsymbol{u}_{n}-\nabla \boldsymbol{u}_{n}^{T}\right): \nabla \boldsymbol{u}_{n} d \boldsymbol{x}=\int_{\Omega} \rho_{n} \mathbf{W}: \nabla \boldsymbol{u}_{n} d \boldsymbol{x}=\frac{1}{2} \int_{\Omega} \rho_{n}\left|\operatorname{rot} \boldsymbol{u}_{n}\right|^{2} d \boldsymbol{x}
$$

we find

$$
\frac{d}{d t}\left(\left\|\sqrt{\rho_{n}} \boldsymbol{u}_{n}\right\|_{L^{2}(\Omega)}^{2}+\kappa\left\|\nabla \rho_{n}\right\|_{L^{2}(\Omega)}^{2}\right)+2 \kappa \lambda\left\|\Delta \rho_{n}\right\|_{L^{2}(\Omega)}^{2}+\frac{\lambda}{2} \int_{\Omega} \rho_{n}\left|\operatorname{rot} \boldsymbol{u}_{n}\right|^{2} d \boldsymbol{x}=2 \int_{\Omega} \rho_{n} \boldsymbol{g} \cdot \boldsymbol{u}_{n} d \boldsymbol{x} .
$$

Since $\boldsymbol{\Delta}=\nabla$ div $-\operatorname{rot}$ rot, we have

$$
\int_{\Omega}\left|\nabla \boldsymbol{u}_{n}\right|^{2} d \boldsymbol{x}=\int_{\Omega}\left|\operatorname{rot} \boldsymbol{u}_{n}\right|^{2} d \boldsymbol{x}
$$

Then, applying the lower bound of $\rho_{n}$ to $\int_{\Omega} \rho_{n}\left|\operatorname{rot} \boldsymbol{u}_{n}\right|^{2} d \boldsymbol{x} \geq m \int_{\Omega}\left|\operatorname{rot} \boldsymbol{u}_{n}\right|^{2} d \boldsymbol{x}$, and the above identity, we obtain

$$
\frac{d}{d t}\left(\left\|\sqrt{\rho_{n}} \boldsymbol{u}_{n}\right\|_{L^{2}(\Omega)}^{2}+\kappa\left\|\nabla \rho_{n}\right\|_{L^{2}(\Omega)}^{2}\right)+2 \kappa \lambda\left\|\Delta \rho_{n}\right\|_{L^{2}(\Omega)}^{2}+\frac{\lambda m}{2}\left\|\nabla \boldsymbol{u}_{n}\right\|_{L^{2}(\Omega)}^{2} \leq 2 \int_{\Omega} \rho_{n} \boldsymbol{g} \cdot \boldsymbol{u}_{n} d \boldsymbol{x} .
$$

In order to estimate the last term, we use Proposition 4.1, the Poincaré and Young inequalities, and we find

$$
2 \int_{\Omega} \rho_{n} \boldsymbol{g} \cdot \boldsymbol{u}_{n} d \boldsymbol{x} \leq \frac{\lambda m}{4}\left\|\nabla \boldsymbol{u}_{n}\right\|_{L^{2}(\Omega)}^{2}+\frac{C M^{2}}{\lambda m}\|\boldsymbol{g}\|_{L^{2}(\Omega)}^{2} .
$$

Finally, we obtain for each $t \in\left[0, T_{n}\right)$

$$
\frac{d}{d t}\left(\left\|\sqrt{\rho_{n}} \boldsymbol{u}_{n}\right\|_{L^{2}(\Omega)}^{2}+\kappa\left\|\nabla \rho_{n}\right\|_{L^{2}(\Omega)}^{2}\right)+2 \kappa \lambda\left\|\Delta \rho_{n}\right\|_{L^{2}(\Omega)}^{2}+\frac{\lambda m}{4}\left\|\nabla \boldsymbol{u}_{n}\right\|_{L^{2}(\Omega)}^{2} \leq \frac{C M^{2}}{\lambda m}\|\boldsymbol{g}\|_{L^{2}(\Omega)}^{2} .
$$

Obviously, thanks to Proposition 5.3, we have the following result:

Corollary $5.4\left(\boldsymbol{u}_{n}, \rho_{n}\right)$ is a global solution of (31) and for all $T>0$,

$$
\begin{aligned}
& \left(\boldsymbol{u}_{n}\right)_{n} \text { is bounded in } L^{\infty}(0, T ; \boldsymbol{H}) \cap L^{2}(0, T ; \boldsymbol{V}), \\
& \left(\rho_{n}\right)_{n} \text { is bounded in } L^{\infty}\left(0, T ; H^{1}(\Omega)\right) \cap L^{2}\left(0, T ; H_{N}^{2}\right) .
\end{aligned}
$$

\subsection{Uniform estimates for time derivatives}

Now, we want to establish uniform estimates for time derivatives $\partial_{t} \rho_{n}$ and $\partial_{t} \boldsymbol{u}_{n}$.

Proposition 5.5 Let $T>0$, then the sequence $\left(\partial_{t} \rho_{n}\right)_{n}$ is bounded in $L^{4 / 3}\left(0, T ; L^{2}(\Omega)\right)$.

Proof. Taking the $L^{2}$-norm of $\partial_{t} \rho_{n}$, using the Hölder and Gagliardo-Nirenberg inequalities and (38), we get

$$
\left\|\partial_{t} \rho_{n}\right\|_{L^{2}(\Omega)} \leq \lambda\left\|\Delta \rho_{n}\right\|_{L^{2}(\Omega)}+C_{0} C_{G N}\left\|\boldsymbol{u}_{n}\right\|_{L^{2}(\Omega)}^{1 / 4}\left\|\nabla \boldsymbol{u}_{n}\right\|_{L^{2}(\Omega)}^{3 / 4}\left\|\rho_{n}\right\|_{L^{\infty}(\Omega)}^{1 / 2}\left\|\Delta \rho_{n}\right\|_{L^{2}(\Omega)}^{1 / 2} .
$$

Using the uniform estimate (42) and (20), we obtain

$$
\left\|\partial_{t} \rho_{n}\right\|_{L^{2}(\Omega)} \leq \lambda\left\|\Delta \rho_{n}\right\|_{L^{2}(\Omega)}+C\left\|\nabla \boldsymbol{u}_{n}\right\|_{L^{2}(\Omega)}^{3 / 4}\left\|\Delta \rho_{n}\right\|_{L^{2}(\Omega)}^{1 / 2} .
$$


Using the Young inequality $a b \leq \frac{1}{2}\left(a^{2}+b^{2}\right)$ in $(49)$, we get

$$
\left\|\partial_{t} \rho_{n}\right\|_{L^{2}(\Omega)} \leq \lambda\left\|\Delta \rho_{n}\right\|_{L^{2}(\Omega)}+C\left\|\nabla \boldsymbol{u}_{n}\right\|_{L^{2}(\Omega)}^{3 / 2}
$$

It is clear that the second hand side in $L^{\frac{4}{3}}(0, T)$ since we have the uniform time estimates $(42)$ and $(43)$ for $\left\|\nabla \boldsymbol{u}_{n}\right\|_{L^{2}(\Omega)}$ and $\left\|\Delta \rho_{n}\right\|_{L^{2}(\Omega)}$ respectively.

Following [4], we establish an estimation of the fractional time derivative of $\boldsymbol{u}_{n}$.

Proposition 5.6 Let $0<\delta<T$ such that

$$
\int_{0}^{T-\delta}\left\|\boldsymbol{u}_{n}(t+\delta)-\boldsymbol{u}_{n}(t)\right\|_{L^{2}(\Omega)}^{2} d t \leq C \delta^{\frac{1}{4}}
$$

with $C$ independent of $n$ and $\delta$.

Proof. For all functions $\phi \in \mathbf{X}_{\mathbf{T}}$, the approximate solution $\left(\boldsymbol{u}_{n}, \rho_{n}\right)$ satisfies the following equation:

$$
\begin{aligned}
& \frac{d}{d \tau} \int_{\Omega} \rho_{n} \boldsymbol{u}_{n} \cdot \boldsymbol{\phi} d \boldsymbol{x}-\int_{\Omega} \rho_{n} \boldsymbol{u}_{n} \cdot \frac{\partial \phi}{\partial \tau} d \boldsymbol{x}-\int_{\Omega} \rho_{n}\left(\boldsymbol{u}_{n} \cdot \nabla\right) \boldsymbol{\phi} \cdot \boldsymbol{u}_{n} d \boldsymbol{x}+\lambda \int_{\Omega}\left(\nabla \rho_{n} \cdot \nabla\right) \boldsymbol{\phi} \cdot \boldsymbol{u}_{n} d \boldsymbol{x} \\
& \quad+\frac{\lambda}{2} \int_{\Omega} \rho_{n} \nabla \boldsymbol{u}_{n}: \nabla \boldsymbol{\phi} d \boldsymbol{x}-\frac{\lambda}{2} \int_{\Omega} \rho_{n} \nabla \boldsymbol{u}_{n}^{T}: \nabla \boldsymbol{\phi} d \boldsymbol{x}=\int_{\Omega} \rho_{n} \boldsymbol{g} \cdot \boldsymbol{\phi} d \boldsymbol{x}-\kappa \int_{\Omega} \Delta \rho_{n} \nabla \rho_{n} \cdot \boldsymbol{\phi} d \boldsymbol{x} .
\end{aligned}
$$

Integrating (52) with respect to $\tau$ between $t$ and $t+\delta$, and taking $\boldsymbol{\phi}=\boldsymbol{u}_{n}(t+\delta)-\boldsymbol{u}_{n}(t)$, we obtain

$$
\begin{aligned}
& \int_{\Omega}\left[\rho_{n}(t+\delta) \boldsymbol{u}_{n}(t+\delta)-\rho_{n}(t) \boldsymbol{u}_{n}(t)\right]\left[\boldsymbol{u}_{n}(t+\delta)-\boldsymbol{u}_{n}(t)\right] d \boldsymbol{x} \\
& \quad=\int_{t}^{t+\delta} \int_{\Omega} \rho_{n}(\tau)\left(\boldsymbol{u}_{n}(\tau) \cdot \nabla\right)\left(\boldsymbol{u}_{n}(t+\delta)-\boldsymbol{u}_{n}(t)\right) \cdot \boldsymbol{u}_{n}(\tau) d \boldsymbol{x} d \tau \\
& \quad-\lambda \int_{t}^{t+\delta} \int_{\Omega}\left(\nabla \rho_{n}(\tau) \cdot \nabla\right)\left(\boldsymbol{u}_{n}(t+\delta)-\boldsymbol{u}_{n}(t)\right) \cdot \boldsymbol{u}_{n}(\tau) d \boldsymbol{x} d \tau \\
& \quad-\frac{\lambda}{2} \int_{t}^{t+\delta} \int_{\Omega} \rho_{n}(\tau) \nabla \boldsymbol{u}_{n}(\tau): \nabla\left(\boldsymbol{u}_{n}(t+\delta)-\boldsymbol{u}_{n}(t)\right) d \boldsymbol{x} d \tau \\
& \quad+\frac{\lambda}{2} \int_{t}^{t+\delta} \int_{\Omega} \rho_{n}(\tau) \nabla \boldsymbol{u}_{n}^{T}(\tau): \nabla\left(\boldsymbol{u}_{n}(t+\delta)-\boldsymbol{u}_{n}(t)\right) d \boldsymbol{x} d \tau \\
& \quad+\int_{t}^{t+\delta} \int_{\Omega} \rho_{n}(\tau) \boldsymbol{g}(\tau) \cdot\left(\boldsymbol{u}_{n}(t+\delta)-\boldsymbol{u}_{n}(t)\right) d \boldsymbol{x} d \tau \\
& \quad-\kappa \int_{t}^{t+\delta} \int_{\Omega} \Delta \rho_{n}(\tau) \nabla \rho_{n}(\tau) \cdot\left(\boldsymbol{u}_{n}(t+\delta)-\boldsymbol{u}_{n}(t)\right) d \boldsymbol{x} d \tau
\end{aligned}
$$

Using the following identity

$$
\rho_{n}(t+\delta) \boldsymbol{u}_{n}(t+\delta)-\rho_{n}(t) \boldsymbol{u}_{n}(t)=\rho_{n}(t+\delta)\left[\boldsymbol{u}_{n}(t+\delta)-\boldsymbol{u}_{n}(t)\right]+\left[\rho_{n}(t+\delta)-\rho_{n}(t)\right] \boldsymbol{u}_{n}(t),
$$


the relation (53) becomes

$$
\begin{aligned}
\| & \sqrt{\rho_{n}(t+\delta)}\left[\boldsymbol{u}_{n}(t+\delta)-\boldsymbol{u}_{n}(t)\right] \|_{L^{2}(\Omega)}^{2} \\
= & -\int_{\Omega}\left[\rho_{n}(t+\delta)-\rho_{n}(t)\right]\left[\boldsymbol{u}_{n}(t+\delta)-\boldsymbol{u}_{n}(t)\right] \cdot \boldsymbol{u}_{n}(t) d \boldsymbol{x} \\
& +\int_{t}^{t+\delta} \int_{\Omega} \rho_{n}(\tau)\left(\boldsymbol{u}_{n}(\tau) \cdot \nabla\right)\left(\boldsymbol{u}_{n}(t+\delta)-\boldsymbol{u}_{n}(t)\right) \cdot \boldsymbol{u}_{n}(\tau) d \boldsymbol{x} d \tau \\
& -\lambda \int_{t}^{t+\delta} \int_{\Omega}\left(\nabla \rho_{n}(\tau) \cdot \nabla\right)\left(\boldsymbol{u}_{n}(t+\delta)-\boldsymbol{u}_{n}(t)\right) \cdot \boldsymbol{u}_{n}(\tau) d \boldsymbol{x} d \tau \\
& -\frac{\lambda}{2} \int_{t}^{t+\delta} \int_{\Omega} \rho_{n}(\tau) \nabla \boldsymbol{u}_{n}(\tau): \nabla\left(\boldsymbol{u}_{n}(t+\delta)-\boldsymbol{u}_{n}(t)\right) d \boldsymbol{x} d \tau \\
& +\frac{\lambda}{2} \int_{t}^{t+\delta} \int_{\Omega} \rho_{n}(\tau) \nabla \boldsymbol{u}_{n}^{T}(\tau): \nabla\left(\boldsymbol{u}_{n}(t+\delta)-\boldsymbol{u}_{n}(t)\right) d \boldsymbol{x} d \tau \\
& +\int_{t}^{t+\delta} \int_{\Omega} \rho_{n}(\tau) \boldsymbol{g}(\tau) \cdot\left(\boldsymbol{u}_{n}(t+\delta)-\boldsymbol{u}_{n}(t)\right) d \boldsymbol{x} d \tau \\
& -\kappa \int_{t}^{t+\delta} \int_{\Omega} \Delta \rho_{n}(\tau) \nabla \rho_{n}(\tau) \cdot\left(\boldsymbol{u}_{n}(t+\delta)-\boldsymbol{u}_{n}(t)\right) d \boldsymbol{x} d \tau \\
= & I_{1}(t)+I_{2}(t)+I_{3}(t)+I_{4}(t)+I_{5}(t)+I_{6}(t)+I_{7}(t) .
\end{aligned}
$$

First, let us estimate $I_{1}(t)$. Using Hölder inequality, we get

$$
\left|I_{1}(t)\right| \leq\left\|\rho_{n}(t+\delta)-\rho_{n}(t)\right\|_{L^{2}(\Omega)}\left\|\boldsymbol{u}_{n}(t+\delta)-\boldsymbol{u}_{n}(t)\right\|_{L^{4}(\Omega)}\left\|\boldsymbol{u}_{n}(t)\right\|_{L^{4}(\Omega)} .
$$

Writing

$$
\rho_{n}(t+\delta)-\rho_{n}(t)=\int_{t}^{t+\delta} \frac{\partial \rho_{n}}{\partial \tau} d \tau
$$

and using the Hölder and Young inequalities and the embedding $H^{1}(\Omega) \subset L^{4}(\Omega)$, we obtain

$$
\left|I_{1}(t)\right| \leq C \delta^{\frac{1}{4}}\left(\int_{t}^{t+\delta}\left\|\frac{\partial \rho_{n}}{\partial \tau}\right\|_{L^{2}(\Omega)}^{\frac{4}{3}} d \tau\right)^{\frac{3}{4}}\left(\left\|\nabla \boldsymbol{u}_{n}(t+\delta)\right\|_{L^{2}(\Omega)}^{2}+\left\|\nabla \boldsymbol{u}_{n}(t)\right\|_{L^{2}(\Omega)}^{2}\right) .
$$

In the same manner, we obtain the following estimations:

$$
\begin{aligned}
& \left|I_{2}(t)\right| \leq C \delta^{\frac{1}{4}}\left(\int_{t}^{t+\delta}\left\|\nabla \boldsymbol{u}_{n}(\tau)\right\|_{L^{2}(\Omega)}^{2} d \tau\right)^{\frac{3}{4}}\left(\left\|\nabla \boldsymbol{u}_{n}(t+\delta)\right\|_{L^{2}(\Omega)}^{2}+\left\|\nabla \boldsymbol{u}_{n}(t)\right\|_{L^{2}(\Omega)}^{2}\right) \\
& \left|I_{3}(t)\right| \leq C \delta^{\frac{3}{8}}\left(\int_{t}^{t+\delta}\left\|\nabla \boldsymbol{u}_{n}(\tau)\right\|_{L^{2}(\Omega)}^{2} d \tau\right)^{\frac{3}{8}}\left(\int_{t}^{t+\delta}\left\|\Delta \rho_{n}(\tau)\right\|_{L^{2}(\Omega)}^{2} d \tau\right)^{\frac{1}{4}}\left(\left\|\nabla \boldsymbol{u}_{n}(t+\delta)\right\|_{L^{2}(\Omega)}^{2}+\left\|\nabla \boldsymbol{u}_{n}(t)\right\|_{L^{2}(\Omega)}^{2}\right)
\end{aligned}
$$

Analogously, one can obtain the desired estimates of $I_{j}(t)$ terms, for $j=4, \ldots, 7$.

Finally, if we choose $0<\delta<1$ and thanks to Propositions 5.3 and 5.5, then by gathering all the above estimates together, we rewrite (54) as follows:

$$
\left\|\sqrt{\rho_{n}(t+\delta)}\left[\boldsymbol{u}_{n}(t+\delta)-\boldsymbol{u}_{n}(t)\right]\right\|_{L^{2}(\Omega)}^{2} \leq C \delta^{\frac{1}{4}}\left(\left\|\nabla \boldsymbol{u}_{n}(t+\delta)\right\|_{L^{2}(\Omega)}^{2}+\left\|\nabla \boldsymbol{u}_{n}(t)\right\|_{L^{2}(\Omega)}^{2}\right) .
$$

Using the lower bound of $\rho_{n}$ and Proposition 5.3, we conclude the proof. 


\subsection{The existence of weak solution $(\boldsymbol{u}, \rho)$}

The final task is to employ the preceding uniform estimates in order to pass to the limit in the approximate problem (31). When $n \rightarrow+\infty$, we have

$$
\boldsymbol{u}_{0 n} \longrightarrow \boldsymbol{u}_{0} \text { in } \mathbf{H} \text { strongly. }
$$

Thanks to (47) and (48), it is possible to choose the subsequences $\left(\boldsymbol{u}_{n}\right)_{n}$ and $\left(\rho_{n}\right)_{n}$ such that

$$
\begin{aligned}
& \boldsymbol{u}_{n} \longrightarrow \boldsymbol{u} \text { in } L^{2}(0, T ; \mathbf{V}) \quad \text { weakly, } \\
& \boldsymbol{u}_{n} \longrightarrow \boldsymbol{u} \text { in } L^{\infty}(0, T ; \mathbf{H}) \quad \text { weakly-star, } \\
& \rho_{n} \quad \longrightarrow \quad \rho \quad \text { in } L^{2}\left(0, T ; H_{N}^{2}\right) \quad \text { weakly, } \\
& \rho_{n} \longrightarrow \rho \quad \text { in } L^{\infty}\left(0, T ; H^{1}(\Omega)\right) \text { weakly-star, } \\
& \partial_{t} \rho_{n} \longrightarrow \partial_{t} \rho \text { in } L^{4 / 3}\left(0, T ; L^{2}(\Omega)\right) \text { weakly. }
\end{aligned}
$$

Using these convergence results, we are able to pass to the limit in the linear terms of (31). Finally, to be able to pass to the limit in the nonlinear terms of (31), it is necessary to use the following strong convergence:

Proposition 5.7 There exists a subsequence $\left(\boldsymbol{u}_{n}, \rho_{n}\right)_{n}$ which converges strongly to $(\boldsymbol{u}, \rho)$ in $L^{2}\left(0, T ; \boldsymbol{L}^{2}(\Omega)\right) \times$ $L^{2}\left(0, T ; H^{1}(\Omega)\right)$. Moreover $(\boldsymbol{u}, \rho)$ is a weak solution of $(1)$.

Proof. Applying some compactness theorems [18, Chap.3, Theorem 2.1] for $\rho_{n}$ and [16, Theorem 5] for $\boldsymbol{u}_{n}$ and using Propositions 5.5 and 5.6, we obtain the desired result.

\section{References}

[1] Antontsev SN, Kazhikhov AV, Monakhov VN. Boundary value problems in mechanics of nonhomogeneous fluids. Studies in Mathematics and Its Applications, 22, North-Holland, Publishing Co., Amesterdam, 1990.

[2] Brenner H. Navier-Stokes revised. Phys. A, 349, 60-132, 2005.

[3] Beirão da Veiga H. Diffusion on viscous fluids. Existence and asymptotic properties of solutions. Annali della Scuola Normale Superiore di Pisa, Classe di Scienze $4^{e}$ série, 10, 2, 341-355, 1983.

[4] Bresch D, Essoufi EH, Sy M. Effects of density dependent viscosities on multiphasic incompressible fluid models. J. Math. Fluid Mech. 9, 3, 377-397, 2007.

[5] Bresch D, Desjardins B, Lin CK. On some compressible fluid models: Korteweg, lubrication and shallow water systems. Comm. Partial Diff. Eqs. 28, 3-4, 843-868, 2003.

[6] Calgaro C, Creusé E, Goudon T. Modeling and simulation of mixture flows: Application to powdersnow avalanches. Computers and Fluids 107, 100-122, 2015.

[7] Dunn JE, Serrin J. On the thermomechanics of interstitial working. Arch. Rational Mech. Anal. 88, 2, 95-133, 1985.

[8] Feireisl E, Novotný A, Petzeltová H. On the existence of globally defined weak solutions to the NavierStokes equations. J. Math. Fluid Mech. 3, 358-392, 2001.

[9] Galdi P, Joseph DD, Preziosi L, Rionero S. Mathematical problems for miscible, incompressible fluids with Korteweg stresses. IMA Preprint Series 702, 1990. 
[10] Joseph DD. Fluid dynamics of two miscible liquids with diffusion and gradient stresses. European J. of Mech. B Fluids 6, 565-596, 1990.

[11] Joseph DD, Huang A, Hu H. Non-solenoidal velocity effects and Korteweg stresses in simple mixture of incompressible liquids. Phys. D, 97, 1-3, 104-125, 1996.

[12] Korteweg DJ. Sur la forme que prennent les équations du mouvement des fluides si l'on tient compte des forces capillaires causées par des variations de densité considérables mais continues et sur la théorie de la capillarité dans l'hypothèse d'une variation continue de la densité. Archives Néerlandaises des Sciences Exactes et Naturelles, Séries II, 6, 1-24, 1901.

[13] Kostin I, Marion M, Texier-Picard R, Volpert VA. Modelling of miscible liquids with the Korteweg stress. M2AN 37, 5, 741-753, 2003.

[14] Kazhikhov A, Smagulov Sh. The correctness of boundary value problems in a diffusion model in an inhomogeneous fluid. Sov. Phys. Dokl. 22, 1, 249-252, 1977.

[15] Lions JL. Quelques méthodes de résolution des problèmes aux limites non linéaires. Dunod, GauthierVillars, Paris, 1969.

[16] Simon J. Compact sets in the space $L^{p}(0, T ; B)$. Ann. Mat. Pura Appl. 146, 65-96, 1987.

[17] Sy M, Bresch D, Guillén-González F, Lemoine J, Rodríguez-Bellido MA. Local strong solution for the incompressible Korteweg model. C. R. Acad. Sci. Paris Ser. I, 342, 169-174, 2006.

[18] Temam R. Navier-Stokes equations, theory and numerical analysis. Revised Edition, Studies in mathematics and its applications vol. 2, North Holland Publishing Company-Amsterdam, New York, 1984. 